\title{
Dust Size Effect On IR Colors Of AGB Stars
}

\author{
Huan Wang, ${ }^{1}$ B. W. Jiang ${ }^{1}$ and R. Szczerba ${ }^{2}$ \\ ${ }^{1}$ Department of Astronomy, Beijing Normal University, Beijing 100875, P.R. China \\ email: whbnu@mail.bnu.edu.cn, bjiang@bnu.edu.cn \\ ${ }^{2}$ Nicolaus Copernicus Astronomical Center, PL-87-100 Torun, Poland \\ email: szczerba@ncac.torun.pl
}

\begin{abstract}
With the Mie theory and the radiative transfer model, we studied the effect of dust size on the infrared color indexes concerning special filters used in the space infrared missions and typical filters in the near-infrared, of AGB stars with typical oxygen-rich and carbon-rich dust shells. It is found the most affected bands are the near-infrared bands JHK and the Spitzer IRAC bands, meanwhile the wavebands with reference wavelength longer than $10 \mu \mathrm{m}$ is little affected. The effect increases fast with the mass loss rate. We also discussed the potential to distinguish the O-rich and C-rich dusts, and the difference in IR colors between the AGB stars and other IR sources like YSOs and galaxies.
\end{abstract}

Keywords. stars: AGB and post-AGB, stars: mass-loss, (stars:) circumstellar matter

\section{Introduction}

The recent launch of space missions such as SPITZER and ASTRO-F boosts our knowledge in the infrared (IR) wave bands of celestial bodies significantly. In the mid-IR wave bands, these missions have such a high sensitivity and spatial resolution that they can detect individual AGB stars in Local Group galaxies. To understand the properties of the AGB stars in local galaxies, the correct explanation of photometric results is necessary. With the optical constants and radiative transfer models, the color indexes related to the special filters in the space missions were calculated by Gronewegen (2006). But with only one dust size of $0.1 \mu \mathrm{m}$, his results needs to be refined since it is established that the circumstellar dust has a wide distribution of size and the size of dust would influence the optical properties and therefore the dust temperature that decides the IR colors.

\section{Calculation and Results}

To study the effect of dust size on the IR colors of AGB stars, the dust opacity is calculated from the Mie theory and the radiative transfer is solved with the dust shells taken into account (Steffen et al., 1997) for a series of dust radius, i.e., $a=0.005,0.01$, 0.035 , and from 0.05 to $0.9 \mu \mathrm{m}$ with a step of $0.05 \mu \mathrm{m}$. The $0.035 \mu \mathrm{m}$ is a reference size, by which the emergent SED is the same as a commonly adopted power-law size distribution of $\mathrm{a}^{-3.5}$ between 0.005 and $0.25 \mu \mathrm{m}$. This size is derived from density-weighted radius and confirmed in the calculation. The species of dust includes $100 \%$ silicate (Laor \& Draine 1993) for an oxygen-rich shell and 100\% amorphous carbon (AMC) (Jaeger et al. 1998) for a carbon-rich shell. To concentrate on the size effect, the stellar parameters are universal and typical for the AGB stars: $L=4390 L_{\odot}, T_{\text {eff }}=3000 \mathrm{~K}, d=8.5 \mathrm{kpc}, \dot{M}=10^{-6} M_{\odot} / \mathrm{yr}$, where the distance refers to the center of the Galaxy and the mass loss rate is typical for an AGB star with moderate mass. The calculated SED is folded with the filters' response for the expected observational magnitude in the corresponding bands. All the filters of the missions which produce abundant data are tested, including 2MASS/JHKs, Spitzer/IRAC/MIPS, Astro-F/IRC/MIR-L/MIR-S/MIR-FIS, IRAS, MSX/ACDE.

It is found that the wave bands affected most by the dust size are the near-IR bands and Spitzer/IRAC bands, while the wavebands with reference wavelength longer than 


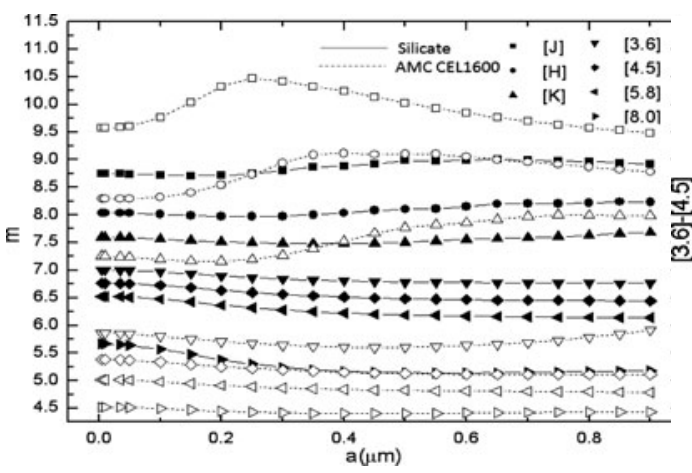

Figure 1.

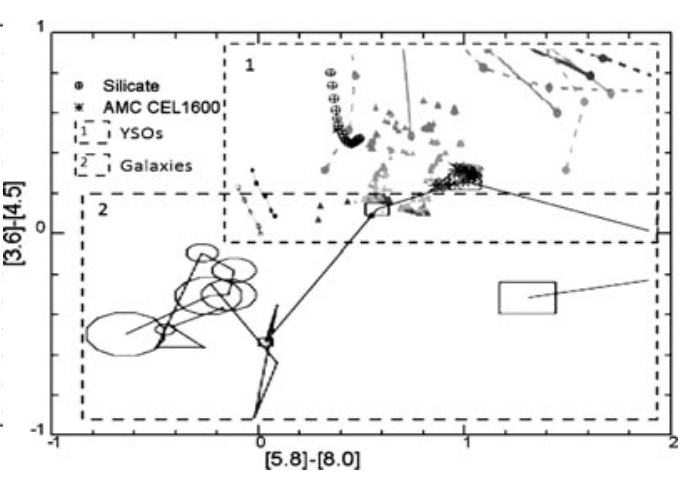

Figure 2.

$10 \mu \mathrm{m}$ are little affected by the dust size in the selected range. This result is consistent with the rule that dust interacts mainly with the radiation at a wavelength comparable to its size, i.e. $\lambda \sim 2 \pi a$. In Figure 1, the change of apparent magnitudes is shown for the wavebands which vary significantly with the dust size. The variations in the JHKs and Spitzer IRAC bands are $0.98 \mathrm{mag}, 0.82 \mathrm{mag}$ and $0.31 \mathrm{mag}$ at J, H and [3.6]. Consequently, the colors related to these bands may vary by similar orders or even larger. It should be pointed out that the order of variation increases fast with the mass loss rate. For a mass loss rate of $5 \times 10^{-6} M_{\odot} / \mathrm{yr}$, the variation with dust size is exceedingly large.

To distinguish the silicate and carbon dust has long been pursued. The colors which have large difference for these two species of dust are J-K, J-H, [5.8]-[8.0] and [3.6]-[4.5].

Another purpose of such calculation is to separate AGB stars from other IR sources, mainly the young stellar objects (YSOs) and extra-galaxies. In Figure 2 are compared the IRAC colors of AGB stars with them, where the colors of YSOs and galaxies are taken from Allen et al. (2004) and Sajina et al. (2005). It can be apparently seen that there is a good separation between the AMC and the silicate dust. Meanwhile the AGB stars can also be separated from the galaxies. But they are mixed with YSOs in particular with thick CSEs in this color-color diagram.

\section{Summary}

(a) The dust size affects the colors related to the wavelength shorter than 10 micron, in particular the near-IR bands such as the JHK and Spitzer/IRAC bands.

(b) The dust size effect increases with the mass loss rate. It is negligible when $\dot{M}<$ $10^{-7} M_{\odot} / \mathrm{yr}$, but increases by $0.6 \mathrm{mag}$ in $\mathrm{J}-\mathrm{K}$ at the rate of $5 \times 10^{-7} M_{\odot} / \mathrm{yr}$.

(c) The effect of O-rich and C-rich dust on the IR colors is different, which provides a potential to distinguish the two chemistry type stars from the IR colors, with the Spitzer/IRAC colors suggested.

(d) The ranges of Spitzer/IRAC color indexes are calculated to be different from extragalaxies.

Acknowledgements: This work is supported by the The Ministry of Science and Technology of the People's republic of China through grant 2007CB815406.

\section{References}

Groenewegen, M. A. T. 2006, AA 448, 181

Steffen, M., Szczerba, R., Menshchikov, A., \& Schoenberner, D. 1997, AApS 126, 39

Laor, A., \& Draine, B. 1993, ApJ 402, 441

Jager, C., Mutschke, H., \& Henning, Th. 1998, AA 332, 291

Allen, L. E., et al. 2004, ApJS 154, 363

Sajina, A., Lacy, M., \& Scott, D. 2005, ApJ 621, 256 